\title{
Isolated Closed Medial Subtalar Dislocation Without Ankle Fracture : A Rare Case Report
}

\author{
Vishwas V Mundewadi ${ }^{1}$, Harshavardhan B R ${ }^{1}$, Akhil Kumar P R ${ }^{1}$
}

\section{Abstract}

Subtalar dislocation is an unusual clinical case with very little mention in literature constituting nearly $1 \%$ of all traumatic dislocations. Here a 40-year-old male who sustained fall over inverted foot was diagnosed as closed isolated medial subtalar dislocation with no associated fractures. This is an even more rare entity. Immediate closed reduction was done and immobilised for 4 weeks with below knee cast. This case report describes the mechanism of injury, ligaments involved, manoeuvre of closed reduction. This case report highlights the importance of prompt and urgent reduction and short period of immobilisation to avoid complications and better prognosis.

Keywords: Subtalar dislocation, Basketball foot, Isolated, Medial, Arthritis.

\section{Introduction}

The isolated subtalar dislocations are extremely rareand this entity is very less mentioned in literature. Here Talocalcaneal and Talonavicular joints are displaced simultaneously. They are usually associated with fractures of Talus, $5^{\text {th }}$ Metatarsa [1], and Malleoli. This constitutes to $1 \%$ of all traumatic dislocations [1]. It was first described by Judey \& Du Faurest in 1811 [2]. In 46-83\% of subtalar dislocation cases it is in the form of open dislocation and in $38.8-88 \%$ cases it is associated with fractures or soft tissue injuries [3].

Broca classified subtalar dislocation into 3 types :- Medial, Lateral, Posterior and Malgaigne \& Henkle modified this to add $4^{\text {th }}$ type Anterior to this [4]. The most common type of subtalar dislocation is Medial (80\%), followed by Lateral (17\%), Anterior and posterior being rare constitutes remaining $3 \%[5,6]$.

Here we report this rare case of 40 -year-old male with history of fall over inverted foot and diagnosed as Isolated closed Medial Subtalar Dislocation. The purpose of this study is to discuss the presentation, diagnosis, conservative treatment and prognosis.

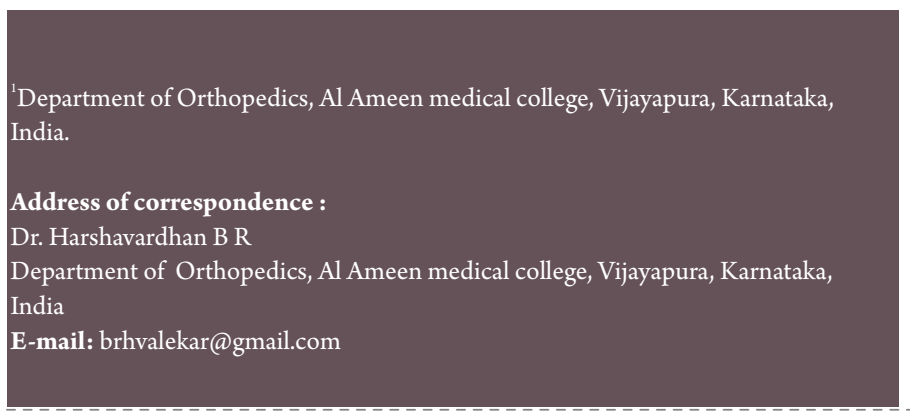

Submitted: 31/6/2021; Reviewed: 19/7/2021; Accepted: 27/7/2021; Published: 10/8/2021

\section{Case Report}

A 40-year-old male patient came to emergency room with complaints of severe pain and inability to move his ankle associated with deformity following fall over inverted foot. The clinical examination revealed diffuse swelling and tenderness over mid and hind foot. Prominent Talus head was palpated dorso-laterally with calcaneum and remaining foot deviated medially in plantar flexion (Figure 1\&2).

Peripheral pulses were normal, no open wound or no sensory deficits. Radiographic examination revealed medial subtalar dislocation with no associated fracture (Figure $3 \& 4$ ). CT scan confirmed the isolated dislocation of talonavicular and talocalcaneal joints with absence of fracture (Figure 5).

Patient was taken to OT and under sedation closed reduction was attempted with Knee in flexion, longitudinal traction was given in line of deformity with mild pressure over talus head and in plantar flexion. Gradually the foot was abducted and everted with simultaneous increase in dorsiflexion. A snap sound was heard with gross correction of deformity (Figure 6 \&7).

The reduction was confirmed by radiographs in AP and Lateral views (Figure $8 \& 9$ ).

A posterior leg splint was used to immobilise as there was swelling. Patient was discharged in stable state and was advised to visit OPD after 3 days. A short leg cast was applied later for 4 weeks with instructions.

Mobilisation protocol was followed with passive and active range of movements once after cast was removed along with partial weight bearing. Full weight bearing was encouraged after 8 weeks of injury. Patient was evaluated in intervals and at the end of 6 months follow up patient had no complaints with which allows others to remix, tweak, and build upon the work non-commercially as long as appropriate credit is given and the new creation are licensed under the identical terms. 


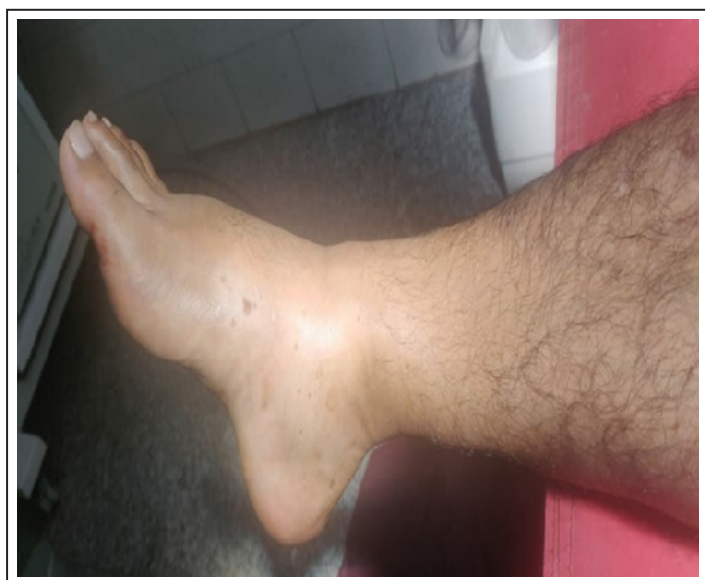

Figure 1: Diffuse swelling and tenderness over mid and hind foot.

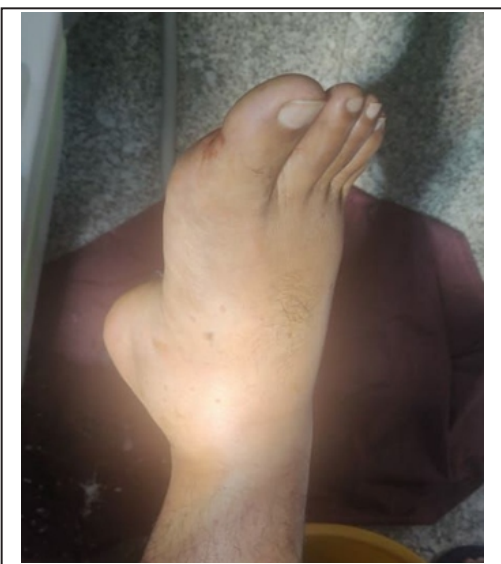

Figure 2: Calcaneum and remaining foot deviated medially in plantar flexion.

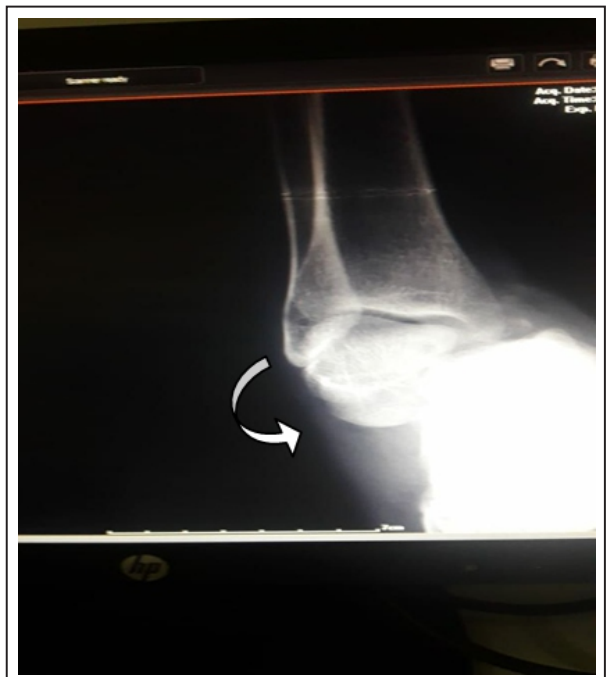

Figure 4: Curved arrow shows medially displaced foot in relation to ankle.

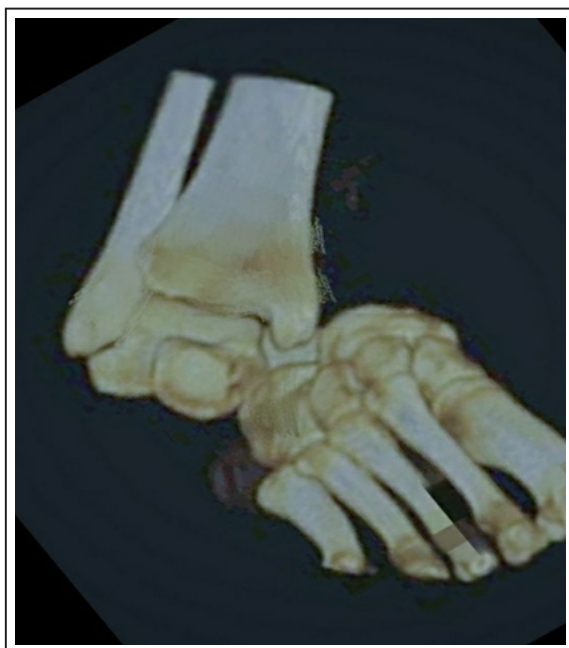

Figure 5: 3D CT scan confirming medial subtalar dislocation without any associated fracture

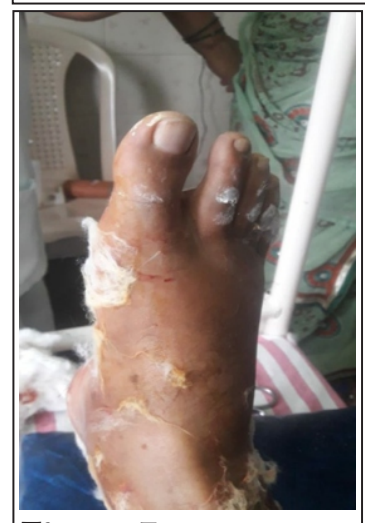

Figure 7: Correction of gross deformity of ankle immediately after closed reduction.

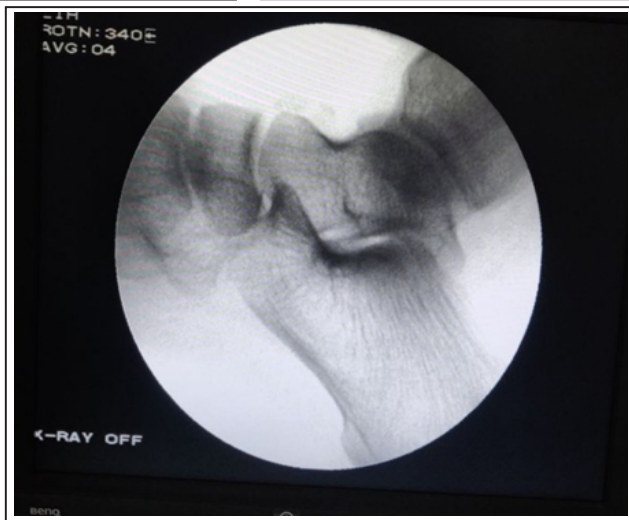

Figure 8: Radiograph of subtalar joint relocated post reduction.
AOFAS score 100 (American Orthopaedic Foot and Ankle Society).

\section{Discussion}

The major etiology behind subtalar dislocation is high energy trauma usually occurred during sports in young males for

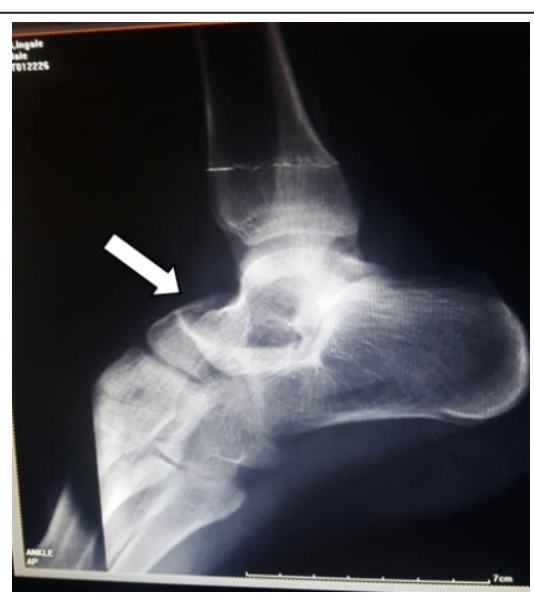

Figure 3: White arrow shows loss of talonavicular Joint alignment

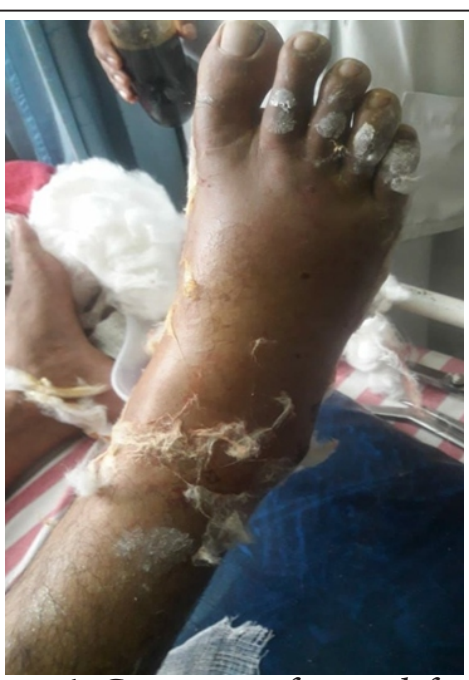

Figure 6: Correction of gross deformity of ankle immediately after closed reduction

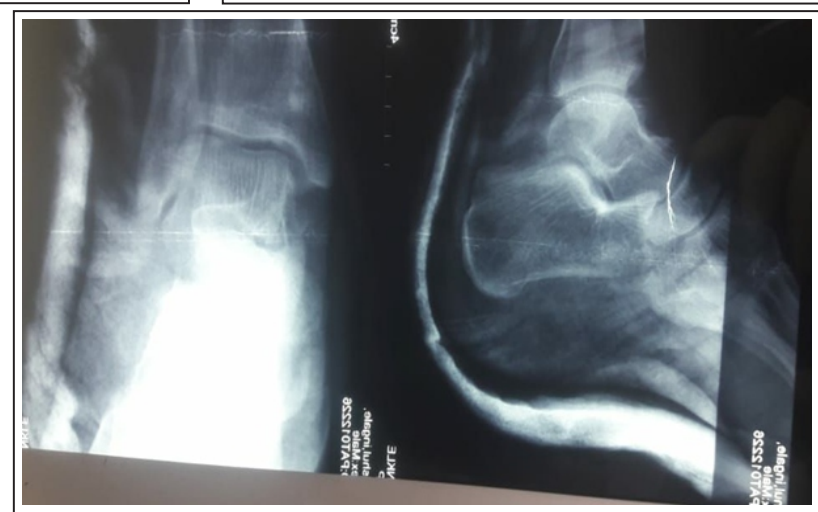

Figure 9: AP \& lateral radiographs of ankle joint post reduction and spilinting. which Grantham termed it as "BASKET BALLFOOT" [7]. Mechanism of injury - when the inversion forces acts on foot, the strong calcaneonavicular ligament bears the stress and resists but the weaker talocalcaneal and talonavicular ligaments give away displacing the entire foot leading to subtalar dislocation. Here the sustentaculum tali acts as fulcrum point 
for medial dislocation [8].

Medial subtalar dislocation is also knows as "ACQUIRED CLUBFOOT" as the foot deviates medially in plantar flexion due to stress over lateral collateral ligament letting the talus be in its normal anatomical position [9]. This internal displacement is explained by the fact that subtalar joint is actually unstable in inversion [10].

Urgent reduction is important and closed reduction under GA/Sedation facilitated by knee in flexion to relax gastrocnemius muscle is usually successful. Sometimes capsule of talonavicular joint and extensor digitorum brevius tendon often block the reduction and open reduction will then be necessary [11].

Excellent prognosis with nearly $100 \%$ success rate is seen in low energy trauma, pure ligamentous dislocations after proper closed reduction. This can be reduced to $15 \%$ in cases resulting from high energy trauma [12]. Merchan reported that $41 \%$ of medical subtalar dislocation cases had cutaneous opening in a series of 39 cases with or without associated fractures. In those, effective debridement and washing of wound was done before

\section{References}

1. Perugia D, Basile A, Massoni C, Gumina S, Rossi F, Ferretti A. Conservative treatment of subtalar dislocations. Int Orthop. 2002;26(1):56-60. doi: 10.1007/s002640100296. PMID: 11954852; PMCID: PMC3620856.

2. Judcy P: Observation d'une luxation métatarsienne. Bulletin de la faculté de medicine. Bull Fac Med Paris. 1811; $11: 81-86$.

3. Prada-Cañizares A, Auñón-Martín I, Vilá Y Rico J, Pretell-Mazzini J. Subtalar dislocation: management and prognosis for an uncommon orthopaedic condition. Int Orthop. 2016 May;40(5):999-1007. doi: 10.1007/s00264 015-2910-8. Epub 2015 Jul 25. PMID: 26208589.

4. Giannoulis D, Papadopoulos DV, Lykissas MG, Koulouvaris P, Gkiatas I, Mavrodontidis A. Subtalar dislocation without associated fractures: Case report and review of literature. World J Orthop. 2015; 6: 374-379 [PMID: 25893182 DOI: 10.5312/wjo.v6.i3.374].

5.Zimmer TJ and Johnson KA. Subtalar Dislocations. Clinical Orthopaedics a n d Re lated Res e a r ch.1989,238, $190-194$. http://dx.doi.org/10.1097/00003086-198901000-00027.

6. Heppenstall RB, Farahvar H, Balderston R. and Lotke P. Evaluation and Management of Subtalar Dislocations. Journal of Trauma. 1980; 20 : 494497. http://dx.doi.org/10.1097/00005373-198006000-00011.

7. Grantham SA, Medial subtalar dislocation: five cases with a common etiology. JTrauma. 1964; vol 4:845-849. PMID: 14225332.

8. Heckman JD. Fractures and dislocations of the foot. In: Rockwood CA Jr, Green DP, Bucholz RW, eds. Fractures in adults. 3rd ed. Philadelphia, etc: JB treating the fracture and increased risk of AVN of talus was found in them [13].

Recommended period of immobilization for isolated closed dislocation is 3-5 weeks and around 6-8 weeks for complicated cases. Immediate rehabilitation and gradual increase in weight bearing favours the functional outcome [14].

\section{Complications}

De Palma et al study suggested correlation between a poor clinical outcome and arthritis of subtalar joint when 30 patients with closed isolated subtalar joint dislocation were reexamined [15].

\section{Conclusion}

Generally Subtalar dislocations are rare and carries serious long term complications like talar necrosis and subtalar arthritis. Although isolated closed medial subtalar dislocation have good prognosis. The key factors for this successful long term outcome is the earliest detection, conservative treatment by closed reduction and carrying prompt follow up protocol.

\section{Lippincott Co.1991;175-89.}

9. Inokuchi S, Hashimoto T, Usami N. Posterior subtalar dislocation. J Trauma. 1997 Feb;42(2):310-3. doi: 10.1097/00005373-199702000-00023. PMID: 9042888 .

10. Jerome JT, Varghese M, Sankaran B. Anteromedial subtalar dislocation. J Foot Ankle Surg. 2007 Jan-Feb;46(1):52-4. doi: 10.1053/j.jfas.2006.10.003. PMID: 17198954.

11. Pua, U. Subtalar dislocation: rare and often forgotten. Int J Emerg Med 2, 51-52 (2009).https://doi.org/10.1007/s12245-009-0086-y

12. Rammelt S, Goronzy J. Subtalar dislocations. Foot Ankle Clin. 2015 Jun;20(2):253-64. doi: 10.1016/j.fcl.2015.02.008. Epub 2015 Mar 29. PMID: 26043242.

13. Merchan EC. Subtalar dislocations: long-term follow-up of 39 cases. Injury. 1992 ; 23(2): 97-100. doi: 10.1016/0020-1383(92)90041-p. PMID: 1572724 .

14. Hoexum F, Heetveld MJ. Subtalar dislocation: two cases requiring surgery and a literature review of the last 25 years. Arch Orthop Trauma Surg. 2014 Sep;134(9):1237-49. doi: 10.1007/s00402-014-2040-6. Epub 2014 Jul 4. PMID: 24993588.

15. de Palma L, Santucci A, Marinelli M, Borgogno E, Catalani A. Clinical outcome of closed isolated subtalar dislocations. Arch Orthop Trauma Surg. 2008 Jun;128(6):593-8. doi: 10.1007/s00402-007-0459-8. Epub 2007 Oct 2.PMID: 17909825.

Declaration of patient consent: The authors certify that they have obtained all appropriate patient consent forms. In the form, the patient has given his consent for his images and other clinical information to be reported in the Journal. The patient understands that his name and initials will not be published, and due efforts will be made to conceal his identity, but anonymity cannot be guaranteed.

\section{Conflict of interest: Nil Source of support: None}

\section{How to Cite this Article}

Mundewadi VV, Harshavardhan BR, Akhil Kumar PR | Isolated Closed Medial Subtalar Dislocation Without Ankle Fracture : A Rare Case Report | Journal of Karnataka Orthopaedic Association | August-September 2021;9(2):15-17. 